\title{
LES VOIES DE LA RÉSILIENCE SE DÉCLINENT À TOUS LES TEMPS DU PASSÉ AU FUTUR
}

\author{
Author(s) / Auteur(s) : \\ Guy KONINCKX \\ Senior Consultant \\ G.I.R.O.S. et C.I.R.E.R.O. \\ guy.koninckx@skynet.be
}

\begin{abstract}
Résumé :
L'effondrement des Twin Towers en septembre 2001, l'éruption volcanique de l'Eyjafjöll en mars 2010 et l'épidémie de maladie à virus Ebola en 2013 ont secoué la planète. La pandémie du COVID-19 ne l'épargne guère et met la planète à l'arrêt.

Très locale au départ, elle s'étend au point de toucher les populations de tous les territoires. Le retentissement affecte le fonctionnement de nombreux domaines. C'est comme si le COVID-19 s'impose dans nos contextes au point de rythmer nos quotidiens aux niveaux macro, meso ou micro.

Formulé en termes systémiques c'est l'ensemble des éléments en interaction dynamique orientés vers une finalité qui se découvrent concernés. Un système s'inscrit dans la temporalité, dans un cycle de vie. Dans le cas présent elle relève d'avantage du cycle de vie d'une crise. Certaines stratégies retenues relèvent des aménagements alors que d'autres relèvent des mutations à opérer. Panser ou penser nous amène à décliner des scénarios différents. Loin de vouloir les opposer purement et simplement, ne s'agit-il pas d'une cohabitation bénéfique? Les enjeux ne se situent-ils pas dans le déploiement de capacités collectives à traverser des crises, de l'amont à l'après, à initier d'autres comportements au sein des systèmes, à revisiter nos croyances?

Ces démarches peuvent s'appuyer sur les ressources de la résilience, les capacités de rebond du système pour naviguer en temps incertains. Il s'agit non seulement de maintenir les activités du système dans des limites acceptables mais de les redéployer dans des activités d'une autre nature. C'est là que la résilience de type 1 ainsi que la résilience de type 2 se complètent et alimentent les temps du décloisonnement.
\end{abstract}

Keywords / Mots-clés :

crise, fonctionnement, niveaux, changement, aménagement, mutation, résiliences

\section{LES CONTEXTES DES VOIES DE LA RÉSILIENCE}

Les imprévus survenus en 2001 aux Twin Towers, l'éruption volcanique de l'Eyjafjöll en 2010 et la transmission de la maladie à virus Ebola en 2013 ont bousculé la planète sans parler de la crise climatique. L'actuelle pandémie de la COVID-19 a enrayé le fonctionnement de la planète depuis fin 2019.

Certains font face et se protègent. D'autres sont atteints et se relèvent, alors que les victimes sont estimées à un million et demi au début décembre 2020. "WHO Coronavirus Disease (COVID-19) Dashboard" [archive], sur covid19.who.int

Pour mémoire la grippe dite espagnole a fait environ 50 millions de victimes en trois vagues successives d'intensités différentes, de 1918 à 1919.

Dans le cas présent, l'ampleur de la crise nous a surpris au point d'imposer le confinement, de fermer nos "systèmes" en quelque sorte.

À ce stade la pandémie met nos systèmes humains en crise et prend le temps de s'installer dans nombre de domaines que ce soit aux niveaux sanitaires, économiques, sociaux, financiers, culturels, psychologiques, organisationnels, politiques et internationaux. De plus elle peut être observée au niveau interne et externe d'une organisation.

Manifestement les organisations, les systèmes dépendent de l'ensemble des circonstances dans lesquelles s'insèrent les relations qui existent entre leurs membres. L'école de Paolo Alto précise que 
les phénomènes, qu'ils soient écologiques, organisationnels, économiques, médicaux ou psychologiques etc ... demeurent partiellement ou totalement incompréhensibles tant que le champ d'observation n'a pas été ouvert suffisamment pour y inclure le contexte. La variable temps est porteuse de l'histoire du système.

$\mathrm{Au}$ cours d'une période de déstabilisation, les repères des individus vacillent au point d'entraîner éventuellement une déconstruction de sens. Potentiellement, ce temps de remous peut aboutir à une rupture ou susciter un rebond. Il s'agit du parcours du choc au rebond. A ce stade, l'observation de comportements initiateurs de résilience s'avère utile. Elle renseigne sur les capacités de rebond au niveau de la personne, de l'équipe et de l'organisation. Celle-ci est susceptible de prendre des formes différentes.

Précisons de quelle manière elle s'inscrit dans le parcours des organisations. Sans être exhaustif il est utile de disposer des caractéristiques de trois moments clés de la crise organisationnelle.

1. l'avant crise ;

2. le pendant la crise ou le temps des turbulences ;

3. l'après crise ou le temps de la rupture.

Disposer de repères peut être utile pour nous permettre de prendre de la hauteur par rapport aux faits. Vient ensuite le moment pour aborder différentes stratégies et issues.

Limiter le concept de résilience au rebond nous semble limitant. C'est pourquoi, afin d'éviter de sérieux raccourcis, il est utile de reprendre deux définitions cadres du concept de résilience.

Un axe majeur des stratégies à déployer consiste à créer des contextes favorables à l'émergence de la résilience. Cette capacité de rebond constitue la propriété d'une organisation qui conserve la même trajectoire ou opte pour une nouvelle voie après une perturbation tout en intégrant certaines transformations.

\section{DÉFINITIONS DU CONCEPT RÉSILIENCE}

Le concept résilience apparaît régulièrement dans les medias. L'assimiler sans plus à rebond est quelque peu réducteur. Le concept a été transposé dans de nombreux domaines. Nous nous limitons aux parcours organisationnels quels que soient leurs tailles, leurs activités et les contextes dans lesquels ils évoluent.

Dans cette optique nous privilégions deux définitions : d'un côté, celle de Stephane Vanistendael et Jacques Lecomte (2000) et Boris Cyrulnik (2001) et, de l'autre, celle de Gilles Teneau et Guy Koninckx $(2010 ; 2016)$.

Les premiers adoptent une définition transversale: "la résilience est la capacité d'une personne ou d'un groupe à se projeter dans l'avenir en dépit d'événements déstabilisants, de conditions de vie difficiles, de traumatismes parfois sévères." (Vanistendael \& Lecomte, 2000)

Les seconds définissent la résilience organisationnelle en tant que capacité d'un système (constitué d'individus travaillant dans une organisation), confronté à des événements fragilisant et déstabilisant, voire catastrophiques, de mobiliser ses ressources en vue de rester en lien avec sa finalité et se projeter dans son futur, en privilégiant l'un des trois scénarios possibles (Teneau \& Koninckx, 2016) :

1. Mettre en place les conditions favorables au processus de résilience avant la crise et sans objectif particulier;

2. Maintenir son activité dans des limites acceptables ;

3. Se redéployer dans des activités d'une autre nature.

Les deux premiers scénarios privilégient des comportements de type homéostatique

Le troisième scénario, en rupture avec les scénarios connus, amène des comportements d'une autre nature. 


\section{LA CONSTRUCTION DU SENS ET RESTER EN LIENS}

Le sens s'apparente à la raison d'être. Il se trouve au cœur des organisations, des équipes ainsi que des individus. Il constitue en quelque sorte la texture de l'organisation. Renouer le passé au présent et au futur revient à mettre en perspective. À l'opposé se profile l'effondrement, le démantèlement, la destruction, l'anéantissement.

Il est utile de le remettre sur le métier au niveau des équipes. Tout comme la résilience le sens se décline à tous les temps.

L'élaboration du sens prend racine dans les interactions ainsi que les liens. Pour Karl E. Weick sa construction relève d'un processus individuel et collectif.

Il s'agit d'extraire de la collection d'événements qui constituent le quotidien de l'organisation.

\section{LES TROIS MOMENTS CLÉS DE LA CRISE OU LE CYCLE DE VIE DE LA CRISE}

La ligne du temps permet d'observer la façon dont évoluent l'intensité de la crise, la perception du sens, l'identité ainsi que la résilience face au choc. Faible avant le choc, le niveau d'intensité de la crise deviendra soutenu pendant la crise avant de baisser. Des signes de perte ou de déconstruction de sens peuvent apparaître. Il en résulte un travail de reconstruction de sens pour la période d'après crise. L'identité suit un parcours similaire. La crise et l'après-crise permettent d'observer les scénarios d'effondrement, de régression, de résilience de types 1 et 2.

Dans un contexte jalonné de perturbations et de crises membres et équipes sont de plus en plus sollicités. Ce qui était stable leur apparaît instable et confus. Il est possible que l'ébauche d'une déconstruction collective de l'identité organisationnelle voit le jour au cours de la crise.

\section{L'AVANT DE LA CRISE}

Des menaces se profilent sur l'organisation. Clairvoyante elle s'organise et se coordonne. Certains la considèrent comme une phase de gestation pendant laquelle des "signaux faibles" se manifestent.

$\mathrm{Au}$ cours de ces circonstances, la situation prend de l'ampleur et des ruptures se profilent au fil des jours. Les personnes sont secouées, elles se réveillent et prennent conscience de la situation.

Une mobilisation se met en place et deux scénarios se dessinent.

Dans le premier scénario, l'organisation est attentive aux événements qui se manifestent dans son environnement. Dans ce cas les membres restent en relation permanente, recueillent un flux d'informations, les traîtent.et y donnent suite. Ils restent en interaction avec l'environnement. C'est le propre des systèmes ouverts.

Dans le second scénario, l'organisation se comporte comme une forteresse assiégée dans laquelle les personnes font fi de l'environnement et refusent de voir la situation. Dans ce cas il s'agit de systèmes fermés. A terme, les systèmes fermés s'isolent au point de ne pas prendre en compte les informations et se mettent en danger. Déconnectés, ils peuvent s'asphyxier. Les interactions se limitent à l'intérieur du système. Les individus se referment sur eux-mêmes et refusent de voir la réalité qui se pointe à l'horizon. Non flexibles, inattentifs et irréfléchis, voire surprotecteurs ils plongent vers des lendemains proches de l'effondrement.

Mettre en place les conditions de résilience de manière proactive constitue un réel atout pour l'organisation.

\section{LE PENDANT LA CRISE OU LE TEMPS DES TURBULENCES}

C'est le temps des incertitudes. Les équipes et l'organisation sont déstabilisées; elles commencent à être déstabilisées. Des problèmes critiques de communications apparaissent. Les enjeux peuvent se révéler considérables. Au dérèglement du fonctionnement s'ajoute les divergences de points de vue éventuels. Tout d'un coup, les équipes se trouvent comme égarées, perdues dans un labyrinthe. Mais, en même temps que cette perturbation, le choc est porteur d'un message d'éveil, il est l'alarme qui fait réagir les équipes et les individus. Cette période de turbulences bouscule les repères, les normes, la 
structure des interactions, etc. La perte de sens s'accentue et crée des conditions favorables à une perte d'identité. Des points de ruptures sont possibles.

Pendant la crise, la mise en place des conditions de résilience s'effectue dans l'urgence.

\section{L'APRÈS CRISE OU LE TEMPS DE LA RUPTURE}

La vision, pourvoyeuse de sens, risque d'être ébranlée pendant l'après crise. Des remises en cause voient le jour. L'avant ne sera pas nécessairement retrouvé. C'est le temps des questionnements voir celui de l'émergence d'un autre monde.

Lorsque la rupture s'observe, il y a altération des références au passé. Des manifestations de la dislocation du sens sont observées et plus rien ne s'apparente au passé.

Patrick Lagadec de dire: "Une rupture est une discontinuité brutale et définitive qui opère par décomposition-recomposition des principes fondateurs les plus essentiels d'un système : visions du monde et missions, repères et valeurs, identités et légitimités, règles du jeu et structures des relations tant en interne qu'avec l'externe, modes de régulation et de communication"

Après cette période de turbulences, le système ne connaît pas toujours un retour à l'équilibre même s'il est différent. De plus, les nécessaires ajustements et changements rencontrent des résistances.

Il est utile d'approfondir l'articulation de la crise et de la résilience.

Le tableau ci-dessous laisse apparaître une synthèse des différences et complémentarités entre la crise et la résilience relevées par Gilles Teneau et Guy Koninckx.

\begin{tabular}{l|l}
\hline \multicolumn{1}{c|}{ Crise } & \multicolumn{1}{c}{ Résilience } \\
\hline Elle se manifeste dans une situation de tension. & $\begin{array}{l}\text { Elle apparaît dans une situation déstabilisante et } \\
\text { traumatisante. }\end{array}$ \\
\hline $\begin{array}{l}\text { Elle est symptomatique. Elle est comme la face visible de } \\
\text { l'iceberg }\end{array}$ & $\begin{array}{l}\text { Elle opère de manière non visible. Elle touche l'univers } \\
\text { émotionnel, les valeurs, les modèles mentaux, } \\
\text { l'engagement. }\end{array}$ \\
\hline Elle provoque un choc et déséquilibre. & $\begin{array}{l}\text { Elle tente de restaurer un équilibre antérieur ou nouveau et } \\
\text { prend la forme d'un rebond. }\end{array}$ \\
\hline $\begin{array}{l}\text { Les repères sont bouleversés. Elle menace et entame le le } \\
\text { sens. }\end{array}$ & Elle revisite ses modèles mentaux et reconstruit du sens. \\
\hline Elle désarticule la réalité. & Elle explore et agence la réalité \\
\hline Elle fragilise les liens. & Elle se construit dans l'interaction et privilégie les liens. \\
\hline Elle est dans l'ici et maintenant. & Elle prend de la distance et favorise la métacommunication. \\
\hline $\begin{array}{l}\text { Latente, diffuse ou explicite, elle connaît un point de de } \\
\text { cristallisation qui se traduit dans un événement. }\end{array}$ & $\begin{array}{l}\text { Elle compose avec le temps et s'élabore à la manière d'un } \\
\text { processus. Elle est évolutive. }\end{array}$ \\
\hline Elle véhicule souvent une connotation négative. & Elle véhicule une connotation positive. Elle revitalise. \\
\hline Elle méconnaît l'identité individuelle et personnelle. & Elle prend appui sur l'identité individuelle et personnelle. \\
\hline Elle menace et entame la confiance. & Elle évolue dans la confiance, l'estime de soi. \\
\hline $\begin{array}{l}\text { Elle provoque des pressions, génère du désarroi et du } \\
\text { stress, amène des résistances. }\end{array}$ & $\begin{array}{l}\text { Elle permet de retrouver progressivement la sérénité et } \\
\text { d'aborder les résistances comme une chance. }\end{array}$ \\
\hline Elle est en décalage avec des ressources internes. & $\begin{array}{l}\text { Les ressources internes des individus, l'auto-efficacité, le } \\
\text { locus de contrôle sont à l'œuvre. }\end{array}$ \\
\hline
\end{tabular}

Table 1. Différences et complémentarités entre crise et résilience (Teneau \& Koninckx, 2010). 


\section{LA RÉSILIENCE ORGANISATIONNELLE ET LE COLLECTIVE SENSMAKING}

Bénédicte Vidaillet dans son étude de Karl Weick ajoute une dimension au cycle de la crise organisationnelle.

"La résilience organisationnelle (Organizational resilience), c'est la capacité d'un groupe à éviter des chocs organisationnels en construisant des systèmes d'action et d'interactions continus destinés à préserver les anticipations des différents individus les uns par rapport aux autres.".

Une organisation n'est pas résiliente en soi; ce sont les membres du système qui sont porteurs de cette capacité. Construire et faire grandir les résiliences individuelles revient à augmenter les capacités organisationnelles. Pour affronter des situations difficiles, Weick souligne l'importance qu'il y a à donner du sens à ce qui est vécu. Il distingue la construction du sens au niveau individuel "sensemaking", de l'élaboration collective du sens, le "collective sensemaking".

Immergé dans le quotidien et pris par les faits, l'individu ne dispose pas de recul pour extraire les éléments de cette "collection d'événements" et les relier. Toutefois c'est au sein d'une représentation qui, en redonnant de l'ordre, donne du sens. L'outil de base incontournable c'est le lien 65.

Pour disposer d'une structure d'interactions favorable, il faut développer sa vigilance et cela, de préférence au niveau collectif. On parlera alors de "vigilance collective"

Plus les individus sont en mesure de considérer et d'accepter des événements inattendus, plus leur champ de perception devient large, plus ils sont collectivement vigilants et préparés à faire face à l'imprévisible. Parmi les caractéristiques favorables à la construction du sens retenons qu'elle prend place dans les interactions entre les membres et implique une tension dynamique entre, d'une part, les processus de reproduction et, d'autre part, les processus d'innovation, d'émergence. De plus, il s'agit d'une activité continue.

L'effondrement de sens se manifeste lorsque l'organisation ne parvient pas à maintenir un système d'interactions organisées et maintient les individus dans des comportements d'inertie.

\section{Les formes de résilience organisationnelle :}

Pour mémoire, voici les trois formes :

- L'effondrement ou forme 1

Dans la mesure où la phase de perte de repères et de sens s'implante, l'absence de rebond se profile. L'énergie fait défaut et le déficit de ressources se solde progressivement par une rupture.

- La résilience de type 1 - Forme 2

C'est l'expression des aptitudes à mobiliser les énergies nécessaires pour retrouver un état d'équilibre antérieur au choc et ce dans des limites acceptables.

- La résilience de type 2 - Forme 3

La résilience de type 2 exprime les aptitudes à mobiliser les énergies nécessaires pour passer la crise et trouver un nouvel équilibre en rupture avec la situation antérieure. Elle envisage de réaliser une bifurcation ou une rupture avec le passé et s'inscrit dans le présent et l'avenir.

\section{REVISITER LES CROYANCES DANS LE CONTEXTE DE LA PANDÉMIE COVID 19}

Voici quelques déclarations :

- Il n'y aura pas de retour à la "normale"

- "Il faut une nouvelle politique de civilisation" déclare Edgard Morin lors d'une interview Le soir 20 avril 2020

- "Il faudra se remettre à vivre mais pas comme avant" ajoute Boris Cyrulnik dans le cadre d'une interview Le soir 31 mars 2020 
- "Détruire la biodiversité, c'est favoriser les épidémies" et "Il faut restaurer les écosystèmes" alerte le WWF

- Jean-Pierre Dupuy invite à voir dans celle-ci un événement nécessaire et possible pour éviter que la catastrophe ne se produise. Selon lui, c'est l'impossibilité de croire que le pire va arriver qui nous empêche d'agir pour qu'elle n'arrive pas.

- "Tout commence toujours par une initiative, une innovation, un nouveau message de caractère déviant, marginal, souvent invisible aux contemporains." (Morin, 2011)

- Il nous faut agir dans le savoir explicite de notre non-savoir Jürgen Habernas

- "J'ai souvent vu qu'en cas de cataclysmes, les solidarités qui étaient endormies pouvaient se réveiller." confie Edgard Morin

\section{CONCLUSIONS ET PERSPECTIVES}

"Ils ne mouraient pas tous mais tous étaient frappés."

Jean de la Fontaine

- Dans un contexte de crise les systèmes non opérants sont en déficit de capacité de résilience. Ils sont vulnérables, se sentent isolés et limités.

- Les capacités de résilience jamais acquises une fois pour toutes sont à entretenir.

- L'échange d'informations à propos des problèmes rencontrés est inspirant pour les systèmes et a fortiori en situation de crise.

- Les résilients sont plus en forme et améliorent leur performance individuelle et collective. Ils sont plus souples, flexibles moins dépendants vis-à-vis de l'organisation. Ils sont plus ouverts.

- Les contextes de crise opèrent comme activateurs de résilience auprès de micro-projets innovants.

- Dans le monde associatif, des utopistes innovent dans des voies contrastant avec les modèles organisationnels classiques : la voie des mutants, précurseurs pour redevenir des vivants..

- La distinction des niveaux micro, meso et macro du système invite à différencier les regards sur l'organisation et fournit des repères d'un autre ordre aux membres. Des interdépendances se font jour.

- La temporalité élargit le scoop des événements : l'avant, le pendant et l'après.

- Imposer un confinement c'est comme si une mise à l'arrêt est induite en contradiction avec les énergies des systèmes ouverts.

- Les interactions saines au sein du système ainsi que les liens sont des contributeurs pour l'élaboration collective de sens.

- L'utilisation des niveaux constitue une boussole bien utile pour déjouer d'éventuelles confusions.

- Le rappel régulier du sens participe à la mobilisation des acteurs du système.

- La vigilance collective constitue une ressource clé pour les trajectoires du système.

- Embarquer pour "Terres inconnues" le monde d'après,

- Opportunité pour nous retrouver avec nous-même. 


\section{RÉFÉRENCES}

CYRULNICK, B. (2001). Les vilains petits canards. Paris, Odile Jacob.

FREUND, J. (1976). Observations sur la dynamique prolégomènes de la crise du conflit. Communications.

HERMESSE, J., LAUGRAND, F., LAURENT, P-J., MAZZOCCHETTE, J., SERVAIS, O., VUILLEMENOT, A-M. (2020). Masquer le monde - Pensées d'anthropologues sur la pandémie. Academia L'Harmattan..

LAGADEC, P., (2000) Ruptures créatrices. Paris, Ed. d'Organisation.

MORIN, E., (2011) La Voie. Fayard.

PIECQ, A. (2011). De la pensée systémique à la pratique de l'organisation. Paris, L'Harmattan.

TENEAU, G \& KONINCKX, G. (2016). "Comprendre la résilience organisationnelle". Odile Jacob, Ionescu, S. Résiliences Ressemblance dans la diversité.

TENEAU, G. \& KONINCKX, G. (2010). Résilience organisationnelle - Rebondir face aux turbulences. Bruxelles, Ed. De Boeck.

TENEAU, G. \& LEMOINE, G. ( 2019). Toxic Handlers Les générateurs de bienveillance. Paris, Odile Jacob.

VANISTENDAEL, S. \& LECOMTE, J. (2000). Le bonheur est toujours possible: Construire la résilience. Paris, Bayard.

VIDAILLET, B. (2003). Le sens de l'action. Paris, Ed. Vuibert.

WATZLAVICK, P., WEAKLAND, J., FISCH, R. (1975). Changements paradoxes et psychothérapies. Paris, Éd. du Seuil.

WEICK, K. (2003). Le sens de l'action. Paris, Ed. Vuiber. 
